\title{
The Cumulative Impact of Sarcopenia, Frailty, Malnutrition, and Cachexia on Other Geriatric Syndromes in Hospitalized Elderly
}

\author{
Doha Rasheedy ${ }^{1 \star}$, Wessam Helmy EL-Kawaly ${ }^{1}$
}

${ }^{1}$ Ain Shams University, Faculty of Medicine, EGYPT

*Corresponding Author: doharasheedy@yahoo.com

Citation: Rasheedy D, EL-Kawaly WH. The Cumulative Impact of Sarcopenia, Frailty, Malnutrition, and Cachexia on Other Geriatric Syndromes in Hospitalized Elderly. Electron J Gen Med. 2021;18(2):em277. https://doi.org/10.29333/ejgm/9695

ARTICLE INFO
Received: 14 Oct. 2020
Accepted: 5 Jan. 2021

Accepted: 5 Jan. 2021

\begin{abstract}
Background: Sarcopenia, frailty, malnutrition, and cachexia are overlapping conditions in hospitalized older adults; they are characterized by altered body composition and are associated with poor outcomes.
\end{abstract}

Aim: to quantify the effect of this overlap on other geriatric giants e.g., delirium, cognitive impairment, and functional disability in hospitalized older adults.

Methods: A cross sectional observational study involving 206 hospitalized older adults was conducted. Each patient was assessed using Arabic validated versions of mini mental state examination, geriatric depression scale15 , the mini nutritional assessment, activities of daily living and instrumental activities of daily living. Furthermore, Johns Hopkins fall risk assessment tool, bioelectrical impedance analysis, handgrip strength, and timed up and go test were performed. Comorbidities were measured using the age adjusted Charlson Comorbidity Index.

Results: $21.4 \%$ of the participants had only one syndrome, $13.1 \%, 14.1 \%$, and $3.4 \%$ had two, three, and four coexisting syndromes, respectively. In the adjusted model for age, gender, and comorbidities, the combined effect of overlapping syndromes was significantly higher than the isolated presence of a single syndrome on the risk of delirium, cognitive impairment, community acquired infections, severe functional disability, high risk of falls, and pressure ulcers.

Conclusions: The overlap between frailty, sarcopenia, malnutrition, and cachexia, increases the risk of many other geriatric giants. The application of the comprehensive geriatric assessment at hospital admission would help clinicians identify this overlap. Moreover, the existing screening protocols for older adults should include these four conditions.

Keywords: aged, cachexia, frailty, sarcopenia, malnutrition

\section{INTRODUCTION}

Sarcopenia, frailty, cachexia, and malnutrition are prevalent health problems in older adults; with loss of body tissues being a major component of each syndrome [1,2]. For many years, these conditions have been extensively studied in parallel [3-7], with great interest paid to the distinction between them. Therefore, many operational definitions were set by different societies to describe each syndrome [8-11]. However, an extensive overlap exists between these definitions and diagnostic criteria [2].

Moreover, these syndromes share similar etiologies, so; they can coexist in the same patient affecting the outcomes and the treatment strategies [1]. Recently, the researchers started to focus on the concurrent occurrence of two or more of these syndromes. The coexistence of sarcopenia and malnutrition [12], sarcopenia, and frailty [13], frailty and malnutrition [14], were all studied in relation to poor outcomes [15].

The coexistence of tissue loss syndromes was recently reported by Sousa-Santos et al., who found that one in every five Portuguese community-dwelling elderly had two or more of sarcopenia, physical frailty, undernutrition, and obesity [16]. Moreover, a recent meta-analysis reported a substantial association between frailty, sarcopenia and malnutrition in hospitalized elderly [17].

The extent to which this overlap increases the risk of other geriatric syndromes e.g., delirium, cognitive impairment, incontinence, physical disability, risk of fall, depression, pressure ulcers, visual deficit, and hearing impairment remains unknown. The aim of this paper was to explore the cumulative impact of sarcopenia, frailty, malnutrition, and cachexia on other geriatric syndromes in hospitalized elderly.

\section{MATERIALS AND METHODS}

\section{Study Design and Population}

This is a cross-sectional study including 206 older adults $(\geq$ 60 years) admitted to the geriatric department of Ain Shams University hospital, Cairo, Egypt, between October 2016 and September 2018. All participants were subjected to 
multidisciplinary comprehensive geriatric assessment (CGA) which included the following:

1. A detailed socio-demographic data, medical history, and physical examination.

2. Cognitive assessment using Arabic mini mental state examination (A-MMSE) [18]. It tests the orientation, registration, attention and calculation, recall, language and praxis. The cutoff for diagnosing cognitive impairment was adjusted for age, gender, and education [18]. A-MMSE was validated for use in Arabic speaking elderly population [19].

3. Assessment of depression was done using Arabic geriatric depression scale-15 (GDS), with scores $\geq 5$ suggested the presence of depression. This Arabic version was validated and exhibited good psychometric properties [20].

4. Nutritional assessment was done using the Arabic Mini Nutritional Assessment (MNA) [21]. It was validated for the assessment of older adults in hospitals, nursing homes, or community. Patients are considered malnourished if $M N A<17$, the risk of malnutrition was diagnosed by MNA score between 17 and 23.5. Good nutritional status was confirmed by MNA $\geq 24$ [22].

5. The premorbid physical function assessment was done using activities of daily living (ADL) [23], and instrumental activities of daily living (IADL) [24]. We used the Arabic versions provided by the Eastern Mediterranean Regional Office of the WHO [25]. Mild disability was diagnosed if the patient had difficulty in transfer and/or shopping or heavy housework. Moderate disability was defined by difficulty in dressing, bathing, or transfer, and/ or preparing meals, doing light housework. Severe disability was diagnosed if a patient had difficulty with eating and/or toileting but not with all ADLs, or difficulty using the telephone and/or managing money but not with all IADLs [26].

6. Comorbidities were assessed using the age adjusted Charlson Comorbidity Index (ACCI), which is a valid tool for predicting the outcome and risk of death using 19 comorbid diseases after adjusting for age [27].

7. Fall risk assessment was done using Johns Hopkins Fall Risk Assessment Tool (JH-FRAT) [28]. It assesses the effect of seven parameters to predict future fall risk [age, previous fall, fecal and urinary incontinence, certain medications, medical equipment (infusion lines, chest tubes, indwelling catheter, etc.), degree of mobility and cognitive status]. The JH-FRAT score $<6$ indicates no risk, low risk 6-13, and high risk $>13$.

8. The objective physical performance measures included handgrip strength (HGS) [29] and timed up and go (TUG) [30].

\section{Definitions of geriatric syndromes}

Delirium was diagnosed according to the Diagnostic and Statistical Manual of Mental Disorders (DSM) 5th edition [31].

Community- acquired infections included those presented on admission or within the next 48 hours of hospital admission. They included community-acquired pneumonia (CAP), urinary tract infections (UTI), acute bronchitis, and cellulitis.

CAP was diagnosed by i) the presence of chest $x$-ray infiltrate on admission; and ii) the presence of one or more major criteria (cough, expectoration, dyspnea, core body temperature $>38.0{ }^{\circ} \mathrm{C}$ ); and iii) Auscultatory findings of abnormal breath sounds and rales [32].

Laboratory confirmed UTI included those with pyuria ( $>10$ $\mathrm{WBC} / \mathrm{mm}^{3}$ per HPF) plus bacteriuria ( $\geq 10^{5} \mathrm{cfu} / \mathrm{mL}$ ) [33].

\section{Definitions of the four syndromes: (Appendix)}

Physical frailty was defined according to the criteria proposed by Fried [8], it includes five domains: weakness, exhaustion, significant weight loss, slowness, and decreased physical activity. While, sarcopenia was defined according to the updated European Working Group on Sarcopenia in Older Persons (EWGSOP) which updated the operational definition of sarcopenia to include the presence of any of the following: low muscle strength, low muscle quantity/quality and low physical performance. Patients can be stratified as having probable, confirmed, or severe sarcopenia [9].

Cachexia was defined by the presence of significant weight loss in the presence of three of the following: decreased muscle strength, anorexia, fatigue, low fat-free mass index, and confirmatory laboratory markers [11].

Malnutrition was defined according to the ESPEN Consensus Statement [10]. Those with MNA SF score below 12 with $\mathrm{BMI}<18.5 \mathrm{~kg} / \mathrm{m} 2$ or significant weight loss combined with either low fat-free mass index or BMI $<20 \mathrm{~kg} / \mathrm{m}^{2}$ if patient's age is less than 70 or BMI $<22 \mathrm{~kg} / \mathrm{m}^{2}$ if age is above 70 years.

Hand-grip strength (HGS) [29], the timed up and go test (TUG) [30], and Muscle mass measurement were performed according to a previously published study [34].

\section{Ethical Consideration}

The study was performed in adherence to the Declaration of Helsinki and the study methodology was approved by the Research Review Board of the Geriatrics and Gerontology Department, Faculty of Medicine, Ain Shams University. Informed consent was obtained from all the participants. However, some of the participants were illiterate and could not provide a signed consent, then verbal consent was documented in the presence of a next of kin and a nurse. The ethics committee approved using of verbal consent.

\section{Sample Size}

Sample was calculated using epiinfo7 (StatCalc) the expected frequency was set at $15-42 \%$ (prevalence of malnutrition and frailty in [1], accepted margin of error $=7 \%$, confidence level $=95 \%$, and $7 \%$ drop out, resulted in target total sample of 206 participants.

\section{Statistical Analysis}

The collected data were analyzed using MedCalc Statistical Software version 18.9.1 (MedCalc Software bvba, Ostend, Belgium; http://www.medcalc.org; 2018). Quantitative variables are presented in the form of means and standard deviation or median and interquartile range. Qualitative variables are presented in the form of frequency tables. Comparison between quantitative variables was carried out using Student $t$ test. Comparison between qualitative variables was carried out using Pearson's $X 2$ test. Mann-Whitney test was used for non-parametric data. Venn diagram was constructed to represent the overlap between tissue loss syndromes. Multivariable logistic regression analysis was used to study the association of tissue loss syndromes accumulation and other geriatric syndromes. Odds ratios (ORs) with 95\% confidence 
Table 1. General description of patients' characteristics stratified by gender

\begin{tabular}{|c|c|c|c|c|c|}
\hline \multicolumn{2}{|c|}{ Variable } & $\begin{array}{c}\text { Total } \\
\text { population } \\
\mathrm{N}=206\end{array}$ & $\begin{array}{l}\text { Women } \\
\mathrm{N}=108\end{array}$ & $\begin{array}{c}\text { Men } \\
\mathrm{N}=98\end{array}$ & $\begin{array}{c}P \\
\text { value }\end{array}$ \\
\hline \multicolumn{2}{|c|}{ Age $($ mean \pm SD) } & $69.45 \pm 7.80$ & $69.58 \pm$ & $69.30 \pm 8.05$ & \\
\hline \multicolumn{2}{|c|}{$\mathrm{ACCl}($ Mean $\pm \mathrm{SD})$} & 1 & 6.39 & $6.04 \pm$ & 58 \\
\hline \multicolumn{2}{|c|}{ BMI (Mean \pm SD) } & 29.43 & 30.93 & 27.93 & 11 \\
\hline \multicolumn{2}{|c|}{ HGS (Mean \pm SD) } & 38.00 & 11.2 & 23.8 & 0.000 \\
\hline \multicolumn{2}{|c|}{ TUG (Mean \pm SD) } & 21.3 & 25.05 & 17.5 & 0.000 \\
\hline \multicolumn{2}{|c|}{ SMI (Mean \pm SD) } & & & & \\
\hline \multicolumn{2}{|c|}{ MMSE (Mean \pm SD) } & 5 & & 25. & \\
\hline \multicolumn{2}{|c|}{ GDS (Mean \pm SD) } & & & & \\
\hline \multicolumn{2}{|c|}{ ADL median (IQR) } & & & & \\
\hline \multicolumn{2}{|c|}{ IADL median (IQR) } & & & & \\
\hline \multicolumn{2}{|c|}{ JH-FRAT median (IQR) } & & & & \\
\hline \multirow{3}{*}{$\begin{array}{l}\text { Fried } \\
\text { criteria }\end{array}$} & Robust & 99( & & & \multirow{3}{*}{0.000} \\
\hline & pref & & & & \\
\hline & frail & & & & \\
\hline \multirow{5}{*}{ EWGSOP } & & & & & \multirow{5}{*}{0.004} \\
\hline & $\begin{array}{c}\text { Pre } \\
\text { sarcopenia }\end{array}$ & $42(20.4 \%)$ & 19(17.6\%) & $23(23.5 \%)$ & \\
\hline & probable & $27(1$ & $18(1$ & 9 & \\
\hline & confirn & & & & \\
\hline & - & & & & \\
\hline \multirow{3}{*}{$\begin{array}{c}\text { Malnutrition } \\
\text { By MNA }\end{array}$} & ivo & & & & \multirow{3}{*}{0.132} \\
\hline & At risk & $63(30.6 \%)$ & $35(32.4 \%)$ & $28(28.6 \%)$ & \\
\hline & malnutrition & $50(24.3 \%)$ & $31(28.7 \%)$ & $19(19.4 \%)$ & \\
\hline \multicolumn{2}{|c|}{ Cachexia } & $28(13.6 \%)$ & $15(13.9 \%)$ & $13(13.3 \%)$ & 0.89 \\
\hline \multicolumn{6}{|c|}{$\begin{array}{l}\text { ACCI: age adjusted Charlson comorbidity index, BMI: body mass index, } \\
\text { HGS: Hand Grip strength, TUG: Timed up and Go test, SMI: skeletal } \\
\text { muscle index, MMSE: mini mental state examination, GDS: Geriatric } \\
\text { depression scale, ADL: Activities of daily living, IADL: Instrumental } \\
\text { activities of daily living, JH-FRAT: Johns Hopkins Fall Risk Assessment } \\
\text { Tool, EWGSOP: updated European Working Group on Sarcopenia in } \\
\text { Older Persons, MNA: mini nutritional assessment }\end{array}$} \\
\hline
\end{tabular}

intervals $(\mathrm{Cl})$ were presented. P-value $<0.05$ was considered to be statistically significant.

\section{RESULTS}

The mean age of the participants was $69.45 \pm 7.80$ years; women comprised $52.4 \%$ of the sample. The mean age adjusted Charlson comorbidity index was $6.22 \pm 1.81$. The prevalence of malnutrition, sarcopenia, frailty, and cachexia were $24.3 \%(n=50), 34.9 \%(n=72), 31.1 \%(n=64)$, and $13.6 \%$ ( $n=28)$, respectively (Table 1 ).

There was statistically significant gender difference in BMI, HGS, TUG, SMI, MMSE, GDS, JH-FRAT, ADL, and IADL. Females were more were more frail and sarcopenic compared to males. Although not reaching statistical significance, women were more at risk for malnutrition.

The most common medical comorbidities were hypertension (HTN) $(59.2 \%, n=122)$, diabetes mellitus (DM) (46.6\%, $n=96)$, and ischemic heart disease (IHD) (37.9\%, $n=78)$, while the most common geriatric giants were functional disability $(49.5 \%, \mathrm{n}=102)$ followed by cognitive impairment (21.4\%, $n=44)$, and depression $(20.5 \%, n=38)$ (Table 2$)$.

DM, HTN, IHD, and thyroid problems were more common among women, while pulmonary diseases were more common among males. Furthermore, cognitive impairment, urine incontinence, immobility, and ADL disability were more prevalent in women (Table 2).
Table 2. Distribution of geriatric syndromes and medical comorbidities stratified by gender

\begin{tabular}{|c|c|c|c|c|c|}
\hline & & $\begin{array}{c}\text { Total } \\
\text { population } \\
\mathrm{N}=206\end{array}$ & $\begin{array}{l}\text { Women } \\
\mathrm{N}=108\end{array}$ & $\begin{array}{l}\text { Men } \\
\mathrm{N}=98\end{array}$ & $\begin{array}{c}P \\
\text { value }\end{array}$ \\
\hline \multicolumn{6}{|c|}{ Medical comorbidities } \\
\hline & DM & $96(46.6 \%)$ & $64(59.3 \%)$ & $32(32.7 \%)$ & 0.000 \\
\hline & HTN & $122(59.2 \%)$ & $78(72.2 \%)$ & $44(44.9 \%)$ & 0.000 \\
\hline & $\mathrm{AF}$ & $33(16.0 \%)$ & $22(20.4 \%)$ & $11(11.2 \%)$ & 0.07 \\
\hline & IHD & $78(37.9 \%)$ & $48(44.4 \%)$ & $30(30.6 \%)$ & 0.04 \\
\hline & art failure & $44(21.4 \%)$ & $18(16.7 \%)$ & $26(26.5 \%)$ & 0.08 \\
\hline & CLD & $42(20.4 \%)$ & $20(18.5 \%)$ & $22(22.4 \%)$ & 0.48 \\
\hline & CKD & $54(26.2 \%)$ & $31(28.7 \%)$ & $23(23.5 \%)$ & 0.39 \\
\hline Gastroin & estinal diseases & $37(18.0 \%)$ & $22(2 C$ & 15 & 0.34 \\
\hline Pulmo & nary diseases & $60(29.1 \%)$ & $16(14.8 \%)$ & $44(44.9 \%)$ & 0.000 \\
\hline \multirow{2}{*}{ Thyroid } & hypothyroidism & $13(6.3 \%)$ & $12(11.1 \%)$ & $1(1 \%)$ & \multirow{2}{*}{-0.007} \\
\hline & hyperthyroidism & $4 \quad 4(1.9 \%)$ & $3(2.8 \%)$ & $1(1 \%)$ & \\
\hline \multicolumn{2}{|c|}{ Parkinsonism } & $5(2.4 \%)$ & $2(1.9 \%)$ & $3(3.1 \%)$ & 0.57 \\
\hline \multicolumn{2}{|r|}{ CVS } & $42(20.4 \%)$ & $28(25.9 \%)$ & $14(14.3 \%)$ & 0.038 \\
\hline \multicolumn{2}{|c|}{ Psychotic disorders } & $3(1.5 \%)$ & $2(1.9 \%)$ & $1(1 \%)$ & 0.61 \\
\hline \multicolumn{2}{|c|}{ anemia } & $64(31.1 \%)$ & $39(36.1 \%)$ & $25(25.5 \%)$ & 0.10 \\
\hline \multicolumn{2}{|c|}{ malignancy } & $11(5.3 \%)$ & $5(4.6 \%)$ & $6(6.1 \%)$ & \\
\hline \multicolumn{6}{|c|}{ Geriatric Syndromes } \\
\hline \multicolumn{2}{|c|}{ Depression } & $38(20.5 \%)$ & $22(23.4 \%)$ & & ) 0.32 \\
\hline \multicolumn{2}{|c|}{ Cognitive impairment } & $44(2$ & $30(2$ & 14 & 0.018 \\
\hline \multicolumn{2}{|c|}{ Incontinence } & $36(17.5 \%)$ & $25(23.1 \%)$ & $11(11.2 \%)$ & 0.024 \\
\hline \multicolumn{2}{|c|}{ Pressure ulcers } & $11(5.3 \%)$ & $8(7.4 \%)$ & $3(3.1 \%)$ & 0.16 \\
\hline \multicolumn{2}{|c|}{ Hearing impairment } & $20(9.7 \%)$ & $10(9.3 \%)$ & $10(10.2 \%)$ & 0.819 \\
\hline \multicolumn{2}{|c|}{ Visual impairment } & $45(21.8 \%)$ & $25(23.1 \%)$ & $20(20.4 \%)$ & 0.635 \\
\hline \multirow{4}{*}{$\begin{array}{c}\text { ADL } \\
\text { Disability }\end{array}$} & No & $104(50.5 \%)$ & $42(38.9 \%)$ & $62(63.3 \%)$ & \multirow{4}{*}{0.005} \\
\hline & $\begin{array}{c}\text { Mild to } \\
\text { moderate }\end{array}$ & $49(23.8 \%)$ & $30(27.8 \%)$ & 19(19.4\%) & \\
\hline & Severe & $38(18.9 \%)$ & $26(24.1 \%)$ & $12(12.2 \%)$ & \\
\hline & Complete & $15(7.3 \%))$ & $10(9.3 \%)$ & $5(5.1 \%)$ & \\
\hline \multirow{3}{*}{ Fall risk } & Low & $130(63.1 \%)$ & $58(53.7 \%)$ & $72(73.5 \%)$ & \multirow{3}{*}{0.012} \\
\hline & moderate & $62(30.1 \%)$ & $40(37.0 \%)$ & $22(22.4 \%)$ & \\
\hline & high & $14(6.8 \%)$ & $10(9.3 \%)$ & $4(4.1 \%)$ & \\
\hline \multicolumn{2}{|c|}{ Immobility } & $22(10.67 \%)$ & $16(14.8 \%)$ & $6(6.1 \%)$ & 0.04 \\
\hline \multicolumn{2}{|c|}{ Delirium } & $38(18.44 \%)$ & $24(22.2 \%)$ & $14(14.3 \%)$ & ) 0.142 \\
\hline \multicolumn{2}{|c|}{$\begin{array}{c}\text { Community acquired } \\
\text { infections }\end{array}$} & $\%)$ & $36(33.3 \%)$ & $27(27.6 \%)$ & 0.368 \\
\hline
\end{tabular}

ADL: activity of daily living, DM: diabetes mellitus, HTN: hypertension, AF: atrial fibrillation, IHD: ischemic heart disease, CLD: chronic liver disease, CKD: chronic kidney disease, CVS: cerebrovascular stroke

The prevalence of community-acquired infections in this study was $62(30.1 \%)$ including $24(11.7 \%)$ UTI, 10 (4.9\%) acute bronchitis, $3(1.5 \%)$ CAP, $3(1.5 \%)$ infected pressure sore, 14 $(6.8 \%)$ cellulitis, $4(1.9 \%)$ had combined UTI, and bronchitis, 1 (0.5\%) septic arthritis, 1 (0.5\%) encephalitis, 1 (0.5\%) diabetic foot, and 1 (0.5\%) typhoid fever (data not shown).

Regarding the prevalence rates of tissue loss syndromes, $21.4 \%$ of the participants had a single syndrome, $13.1 \%, 14.1 \%$, and $3.4 \%$ had two, three, and four coexisting tissue loss syndromes, respectively (Figure 1).

Table 3 showed that, the prevalence of cognitive impairment, delirium, pressure ulcer, community acquired infections, severe disability, and high risk of falls increased progressively with the increased number of coexisting tissue loss syndromes.

When both genders were analyzed separately, the prevalence of cognitive impairment, delirium, $A D L$ dependency, and high risk of falls increased progressively with the increased number of coexisting tissue loss syndromes in both genders. In males, the prevalence of urinary incontinence and community acquired infections increased with 
Venn Diagram

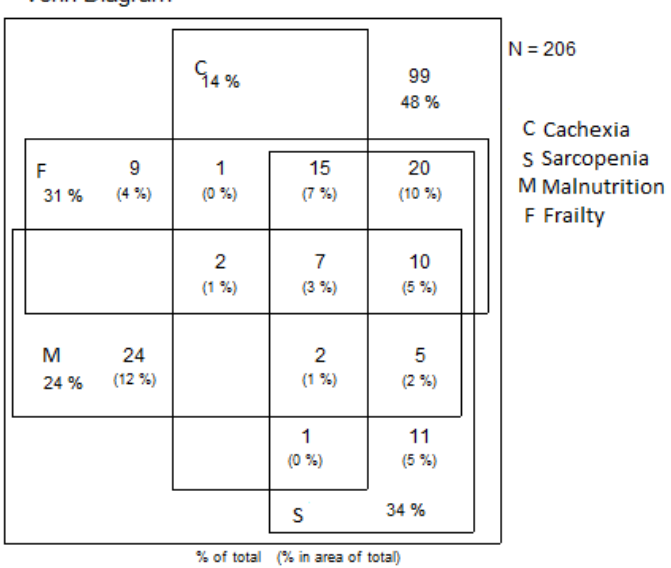

Figure 1. Overlap of sarcopenia, frailty, cachexia, and malnutrition in older medical inpatients $(n=206)$ accumulation of the four conditions. In women, there was significant increase in the frequency of pressure ulcers with the increased number of coexisting tissue loss syndromes (Table 3).

In the adjusted model for age, gender, and $\mathrm{ACCl}$, the increased number of coexisting tissue loss syndromes increased the risk of delirium, cognitive impairment, community acquired infections, severe functional disability, high risk of falls, and pressure ulcer development (Table 4).

The presence of one, two, three or more of the tissue loss syndromes had $(\mathrm{OR}=3.29,95 \% \mathrm{Cl} 1.11-9.77, \mathrm{P}=0.03),(\mathrm{OR}=$ $4.24,95 \% \mathrm{Cl} 1.31-13.72, \mathrm{P}=0.0005)$, and $(\mathrm{OR}=9.25,95 \% \mathrm{Cl} 3.13$ - 27.29, $P=0.0001$ ) for having delirium.

Similarly, the presence of one, two, three or more of the tissue loss syndromes had $(\mathrm{OR}=4.23,95 \% \mathrm{Cl} 1.29-13.88, \mathrm{P}=$

Table 3. Effect of overlapping of the four syndromes on other geriatric syndromes

\begin{tabular}{|c|c|c|c|c|c|c|}
\hline \multirow{2}{*}{\multicolumn{2}{|c|}{ Geriatric syndromes }} & \multicolumn{4}{|c|}{ Number of Syndromes with body tissue loss in total sample } & \multirow{2}{*}{ P value } \\
\hline & & Absent ( $\mathrm{N}=99)$ & 1 syndrome $(\mathrm{N}=44)$ & 2 syndromes $(\mathrm{N}=27)$ & 3 or 4 syndromes $(\mathrm{N}=36)$ & \\
\hline \multicolumn{2}{|c|}{ Cognitive impairment } & $5(5.1 \%)$ & $9(20.5 \%)$ & $8(29.6 \%)$ & $22(61.1 \%)$ & $0.000^{\star}$ \\
\hline \multicolumn{2}{|c|}{ Depression } & $15(15.6 \%)$ & $11(26.2 \%)$ & $4(18.2 \%)$ & $8(32.0 \%)$ & 0.228 \\
\hline \multicolumn{2}{|c|}{ Delirium } & $7(7.1 \%)$ & $9(20.5 \%)$ & $7(25.9 \%)$ & $15(41.7 \%)$ & $0.000^{\star}$ \\
\hline \multirow{3}{*}{$\begin{array}{c}\text { Functional } \\
\text { disability }\end{array}$} & No & $76(76.8 \%)$ & $22(50.0 \%)$ & $5(18.5 \%)$ & $1(2.8 \%)$ & \multirow{3}{*}{$0.000^{\star}$} \\
\hline & Mild to moderate & $19(19.2 \%)$ & $14(31.8 \%)$ & $9(33.3 \%)$ & $7(19.4 \%)$ & \\
\hline & Severe to complete & $4(4.0 \%)$ & $8(18.2 \%)$ & $13(48.1 \%)$ & $28(77.8 \%)$ & \\
\hline \multicolumn{2}{|c|}{ Urinary incontinence } & $10(10.1 \%)$ & $10(22.7 \%)$ & $8(29.6 \%)$ & $8(22.2 \%)$ & 0.048 \\
\hline \multicolumn{2}{|c|}{ Pressure ulcer } & $1(1.0 \%)$ & $1(2.3 \%)$ & $2(7.4 \%)$ & $7(19.4 \%)$ & $0.000^{*}$ \\
\hline \multicolumn{2}{|c|}{ Hearing impairment } & $9(9.1 \%)$ & $6(13.6 \%)$ & $1(3.7 \%)$ & $4(11.1 \%)$ & 0.57 \\
\hline \multicolumn{2}{|c|}{ Visual impairment } & $25(25.3 \%)$ & $8(18.2 \%)$ & $5(18.5 \%)$ & $7(19.4 \%)$ & 0.725 \\
\hline \multicolumn{2}{|c|}{ Community acquired infections } & $19(19.2 \%)$ & $15(34.1 \%)$ & $11(40.7 \%)$ & $18(50 \%)$ & $0.003^{*}$ \\
\hline \multirow{3}{*}{ Fall risk } & low & $80(80.8 \%)$ & $24(54.5 \%)$ & $10(37.0 \%)$ & $15(41.7 \%)$ & \multirow{3}{*}{$0.000^{\star}$} \\
\hline & moderate & $17(17.2 \%)$ & $17(38.6 \%)$ & $14(51.9 \%)$ & $14(38.9 \%)$ & \\
\hline & high & $2(2.0 \%)$ & $3(6.8 \%)$ & $3(11.1 \%)$ & $7(19.4 \%)$ & \\
\hline \multicolumn{2}{|c|}{ females } & \multicolumn{4}{|c|}{ Number of Syndromes with body tissue loss in women } & \multirow{2}{*}{ P value } \\
\hline \multicolumn{2}{|c|}{ Geriatric syndromes } & Absent ( $\mathrm{N}=39)$ & 1 syndrome $(\mathrm{N}=29)$ & 2 syndromes $(\mathrm{N}=17)$ & 3 or 4 syndromes $(\mathrm{N}=23)$ & \\
\hline \multicolumn{2}{|c|}{ Cognitive impairment } & $3(7.7 \%)$ & $7(24.1 \%)$ & $4(23.5 \%)$ & $16(69.6 \%)$ & $0.000^{*}$ \\
\hline \multicolumn{2}{|c|}{ Depression } & $8(20.5 \%)$ & $8(29.6 \%)$ & $1(6.7 \%)$ & $5(38.5 \%)$ & 0.19 \\
\hline \multicolumn{2}{|c|}{ Delirium } & $4(10.3 \%)$ & $5(17.2 \%)$ & $5(29.4 \%)$ & $10(43.5 \%)$ & $0.017^{\star}$ \\
\hline & No & $26(66.7 \%)$ & $12(41.4 \%)$ & $4(23.5 \%)$ & 0 & \\
\hline $\begin{array}{l}\text { ADL } \\
\text { denendency }\end{array}$ & Mild to moderate & $11(28.2 \%)$ & $11(37.9 \%)$ & $4(23.5 \%)$ & $4(17.4 \%)$ & $0.000^{\star}$ \\
\hline & Severe to complete & $2(5.1 \%)$ & $6(20.7 \%)$ & $9(52.9 \%)$ & $19(82.6 \%)$ & \\
\hline Urinar & incontinence & $7(17.9 \%)$ & $8(29.6 \%)$ & $4(23.5 \%)$ & $6(26.1 \%)$ & 0.79 \\
\hline Pre & sure ulcer & 0 & $1(3.4 \%)$ & $1(5.8 \%)$ & $6(26.1 \%)$ & $0.001^{\star}$ \\
\hline Hearin & impairment & $3(7.7 \%)$ & $4(13.8 \%)$ & $1(5.8 \%)$ & $2(8.7 \%)$ & 0.78 \\
\hline Visua & impairment & $11(28.2 \%)$ & $7(24.1 \%)$ & $2(11.8 \%)$ & $5(38.5 \%)$ & 0.60 \\
\hline Community & cquired infections & $10(25.6 \%)$ & $10(34.5 \%)$ & $6(35.3 \%)$ & $10(43.5 \%)$ & 0.54 \\
\hline & Low & $27(69.9 \%)$ & $14(48.3 \%)$ & $6(35.3 \%)$ & $11(47.8 \%)$ & \\
\hline Fall risk & Moderate & $12(30.8 \%)$ & $13(44.8 \%)$ & $8(47.1 \%)$ & $7(30.4 \%)$ & $0.033^{*}$ \\
\hline & High & 0 & $2(6.9 \%)$ & $3(17.6 \%)$ & $5(21.7 \%)$ & \\
\hline & Males & & Number of Syndrom & with body tissue los & $s$ in men & \\
\hline Geriat & c syndromes & Absent $(\mathrm{N}=60)$ & 1 syndrome $(\mathrm{N}=15)$ & 2 syndromes $(\mathrm{N}=10)$ & 3 or 4 syndromes $(\mathrm{N}=13)$ & Pvalue \\
\hline Cogniti & e impairment & $2(3.3 \%)$ & $2(13.3 \%)$ & $4(40 \%)$ & $6(46.2 \%)$ & $0.000^{*}$ \\
\hline & pression & $7(12.3 \%)$ & $3(20 \%)$ & $3(30 \%)$ & $3(23.1 \%)$ & 0.19 \\
\hline & elirium & $3(5 \%)$ & $4(26.7 \%)$ & $2(20 \%)$ & $5(38.5 \%)$ & $0.006^{\star}$ \\
\hline & No & $50(83.3 \%)$ & $10(66.7 \%)$ & $1(10 \%)$ & $1(7.7 \%)$ & \\
\hline ADL & Mild to moderate & $8(13.3 \%)$ & $3(20 \%)$ & $5(50 \%)$ & $3(23.1 \%)$ & $0.000^{\star}$ \\
\hline & Severe to complete & $2(3.3 \%)$ & $2(13.3 \%)$ & $4(40 \%)$ & $9(69.2 \%)$ & \\
\hline Urinar & incontinence & $3(5 \%)$ & $2(13.3 \%)$ & $4(40 \%)$ & $2(15.4 \%)$ & $0.012^{*}$ \\
\hline Pre & sure ulcer & $1(1.7 \%)$ & 0 & $1(10 \%)$ & $1(7.7 \%)$ & 0.33 \\
\hline Hearin & impairment & $6(10 \%)$ & $2(13.3 \%)$ & 0 & $2(15.4 \%)$ & 0.64 \\
\hline Visua & impairment & $14(23.2 \%)$ & $1(6.7 \%)$ & $3(30 \%)$ & $2(15.4 \%)$ & 0.41 \\
\hline Community & cquired infections & $9(15 \%)$ & $5(33.3 \%)$ & $5(50 \%)$ & $8(61.5 \%)$ & $0.002^{*}$ \\
\hline & Low & $53(88.3 \%$ & $10(66.7 \%)$ & $4(40 \%)$ & $4(30.8 \%)$ & \\
\hline Fall risk & Moderate & $5(8.3 \%)$ & $4(26.7 \%)$ & $6(60 \%)$ & $7(53.8 \%)$ & $0.000^{\star}$ \\
\hline & High & $2(3.3 \%)$ & $1(6.7 \%)$ & 0 & $2(15.4 \%)$ & \\
\hline
\end{tabular}


Table 4. Adjusted OR and 95\% $\mathrm{Cl}$ for the accumulated effect of the tissue loss syndromes for other geriatric syndromes

\begin{tabular}{|c|c|c|c|c|c|}
\hline Geriatric giant & Number of tissue loss syndromes & $\beta$ coefficient & Odds ratio & $95 \% \mathrm{Cl}$ & P value \\
\hline \multirow{3}{*}{ Delirium } & One syndrome & 1.19 & 3.29 & $1.11-9.77$ & $0.03^{\star}$ \\
\hline & Two syndromes & 1.44 & 4.24 & $1.31-13.72$ & $0.01^{\star}$ \\
\hline & $\geq$ Three syndromes & 2.22 & 9.25 & $3.13-27.29$ & $0.0001^{\star}$ \\
\hline \multirow{3}{*}{ Cognitive impairment } & One syndrome & 1.44 & 4.23 & $1.29-13.88$ & $0.01^{\star}$ \\
\hline & Two syndromes & 1.87 & 6.51 & $1.87-22.65$ & $0.003^{\star}$ \\
\hline & Three syndromes & 3.17 & 23.85 & $7.39-76.97$ & $<0.0001^{\star}$ \\
\hline \multirow{3}{*}{$\begin{array}{l}\text { Community acquired } \\
\text { Infections }\end{array}$} & One syndrome & 0.81 & 2.24 & $0.98-5.12$ & 0.053 \\
\hline & Two syndromes & 1.05 & 2.86 & $1.12-7.28$ & $0.02^{\star}$ \\
\hline & $\geq$ Three syndromes & 1.51 & 4.56 & $1.88-11.07$ & $0.0008^{\star}$ \\
\hline \multirow{3}{*}{$\begin{array}{c}\text { Severe - complete } \\
\text { disability }\end{array}$} & One syndrome & 1.55 & 4.71 & $1.28-16.78$ & $0.017^{\star}$ \\
\hline & Two syndromes & 2.97 & 19.49 & $5.48-69.33$ & $<0.0001^{\star}$ \\
\hline & $\geq$ Three syndromes & 4.38 & 58.72 & $15.17-227.30$ & $<0.0001^{*}$ \\
\hline \multirow{3}{*}{ High fall risk } & One syndrome & 1.26 & 3.88 & $0.61-24.45$ & 0.14 \\
\hline & Two syndromes & 1.67 & 5.80 & $0.90-37.09$ & 0.063 \\
\hline & $\geq$ Three syndromes & 2.51 & 4.88 & $1.64-14.51$ & $0.004^{\star}$ \\
\hline \multirow{3}{*}{ Pressure ulcers } & One syndrome & 0.56 & 2.06 & $0.12-33.95$ & 0.61 \\
\hline & Two syndromes & 1.87 & 7.66 & $0.65-89.82$ & 0.10 \\
\hline & $\geq$ Three syndromes & 2.69 & 10.01 & $2.75-36.40$ & $0.0005^{\star}$ \\
\hline \multirow{3}{*}{ Urinary incontinence } & One syndrome & 0.73273 & 2.0808 & 0.7669 to 5.6455 & 0.1502 \\
\hline & Two syndromes & 1.08715 & 2.9658 & 0.9865 to 8.9160 & 0.0529 \\
\hline & $\geq$ Three syndromes & 0.40495 & 1.4992 & 0.5003 to 4.4924 & 0.4695 \\
\hline
\end{tabular}

0.01), $(\mathrm{OR}=6.51,95 \% \mathrm{Cl} 1.87-22.65, \mathrm{P}=0.003)$, and $(\mathrm{OR}=23.85$, $95 \% \mathrm{Cl} 7.39$ - 76.97, $\mathrm{P}<0.0001$ ) for cognitive impairment.

The presence of 3 or more tissue loss syndromes was associated with severe disability with $(\mathrm{OR}=58.72,95 \% \mathrm{Cl} 15.17$ -227.30, $\mathrm{P}=<0.0001$ ), high fall risk as assessed by JH-FRAT (OR $=4.88,95 \% \mathrm{Cl} 1.64-14.51, \mathrm{P}=0.0004)$, pressure sores $(\mathrm{OR}=$ $10.01,95 \% \mathrm{Cl} 2.75-36.40, \mathrm{P}=0.0005)$, and infections ( $\mathrm{OR}=4.56$, $95 \% 1.88-11.07, \mathrm{P}=0.0008$ ) (Table 4).

\section{DISCUSSION}

To our knowledge, the prevalence of concurrent occurrence of these syndromes in older inpatients were extensively studied, yet, this is the first study to address the impact of this overlap on other geriatric syndromes including delirium, cognitive impairment, incontinence, physical disability, risk of fall, depression, pressure ulcers, visual deficit, and hearing impairment.

The current work showed that at least one of the tissue loss syndromes was present in $51.9 \%$ of the studied sample. This prevalence was lower than a previous study by Gingrich and colleagues [1], who reported that $63 \%$ of the participants had at least one syndrome. This difference is mainly attributed to higher prevalence of oncological diseases in their study (31\%).

In this study, $21.4 \%$ of the participants had only one syndrome, $13.1 \%, 14.1 \%$, and $3.4 \%$ had two, three, and four coexisting tissue loss syndromes, respectively. According to Gingrich and colleagues [1], 32\% of patients had one tissue loss syndrome, $11 \%$ had two, $12 \%$ had three and $8 \%$ had all four.

The overlap between frailty and sarcopenia was the most common overlap among the studied population. This overlap accounted for $25.3 \%$ of the cases. Sarcopenia was the most common condition followed by frailty; they accounted for $34.9 \%(n=72), 31.1 \%(n=64)$, respectively.

Sarcopenia is primarily diagnosed by low physical performance; making sarcopenia one of the components used to define physical frailty. This indicates a considerable overlap between frailty and sarcopenia in different clinical settings.
[35] Many previous studies documented their overlap $[16,17,37,38]$.

Malnutrition co-occurred with sarcopenia and frailty in $11.7 \%$ and $9.3 \%$ of the studied sample, respectively. Malnutrition plays a key role in the pathogenesis of both frailty [39] and sarcopenia [40,41]. Thus, nutritional interventions should be an integral part of sarcopenia and frailty prevention and treatment programs.

To the best of our knowledge, this is the first study addressing the cumulative effect of these conditions on other geriatric giants.

The prevalence of cognitive impairment, delirium, pressure ulcer, community acquired infections, severe disability, and high risk of falls increased with the increased number of coexisting tissue loss syndromes in older inpatients. This effect persisted after adjustment of other cofounding variables i.e. age, gender, and medical comorbidities. However, the prevalence of depression, visual and hearing disabilities was not affected by the combined effect of tissue loss syndromes. This is probably due to the increased prevalence of these conditions even in the absence of the tissue loss syndromes.

Many studies have been conducted to evaluate the negative consequences of each tissue loss syndrome. The associations of cognitive decline and frailty [42], sarcopenia [38], and malnutrition were reported [43]. Similar data was found linking delirium to frailty [44], sarcopenia [45], and malnutrition in frail older adults [46].

Moreover, a recent study reported that the history of recurrent falls was higher among older adults having both sarcopenia and frailty compared to the robust, the sarcopenia only and the frailty only groups [47].

Our findings indicated that the four tissue loss syndromes could be considered a cluster of risk factors for many other geriatric giants. This approach of addressing the tissue loss syndromes as a cluster better suits recent trends in aging research, focusing on multi-morbidity and coexisting conditions $[48,49]$. Thus, the preventive and therapeutic interventions should be designed to target this overlap. 
There were several limitations to this study. First, the crosssectional design limits the implications of any causal reasoning between the overlapping tissue loss syndromes and other geriatric giants.

Second, the relatively small sample size and being conducted at a single-center may reduce the power of analysis, and limit generalization. However, this sample size is larger than previous studies investigating the overlap of tissue loss syndromes in hospital settings [1].

Third, the study was performed in hospital settings, thus results can't be generalized to older adults in outpatient settings.

Fourth, future longitudinal studies are required to clarify the significance of single and concurrent occurrence of these syndromes on clinical outcomes, length of hospital stay, and mortality.

\section{CONCLUSIONS}

The tissue loss syndromes (sarcopenia, frailty, cachexia, and malnutrition) were common among older medical inpatients. These syndromes overlapped and occurred concurrently. There is a substantial synergistic effect of multiple tissue loss syndromes on the risk of other geriatric giants including delirium, cognitive impairment, risk of fall, functional disability, and pressure ulcers.

\section{CLINICAL IMPLICATIONS}

- Owing to the considerable overlap between sarcopenia, frailty, cachexia, and malnutrition among hospitalized elderly, health-care providers should focus on identifying this overlap by administrating comprehensive assessment upon admission.

- Providing multimodal therapeutic intervention (nutrition and exercise) should be initiated upon diagnosing any of these conditions followed by monitoring for subsequent occurrence of other syndromes.

- Co-occurrence of any of these syndrome should alert physician to screen for other geriatric giants like cognitive impairment, risk of fall, functional limitation.

Author contributions: All authors have sufficiently contributed to the study, and agreed with the results and conclusions.

Funding: No funding source is reported for this study.

Declaration of interest: No conflict of interest is declared by authors. Acknowledgements: The authors acknowledge all the participants in the study.

\section{REFERENCES}

1. Gingrich A, Volkert D, Kiesswetter E, Thomanek M, Bach S, Sieber CC, et al. Prevalence and overlap of sarcopenia, frailty, cachexia and malnutrition in older medical inpatients. BMC Geriatr. 2019 Dec;19(1):120. https://doi.org/10.1186/s12877-019-1115-1 PMid:31029082 PMCid:PMC6487020
2. Jeejeebhoy KN. Malnutrition, fatigue, frailty, vulnerability, sarcopenia and cachexia: overlap of clinical features. Current Opinion in Clinical Nutrition and Metabolic Care. 2012 May;15(3):213-9. https://doi.org/10.1097/MCO. 0b013e328352694f PMid:22450775

3. Morley JE, Anker SD, von Haehling S. Prevalence, incidence, and clinical impact of sarcopenia: facts, numbers, and epidemiology-update 2014. J Cachexia Sarcopenia Muscle. 2014 Dec;5(4):253-9. https://doi.org/10.1007/s13539-0140161-y PMid:25425503 PMCid:PMC4248415

4. Clegg A, Young J, Iliffe S, Rikkert MO, Rockwood K. Frailty in elderly people. The Lancet. 2013 Mar;381(9868):752-62. https://doi.org/10.1016/S0140-6736(12)62167-9

5. Morley JE, Silver AJ. Anorexia in the elderly. Neurobiology of Aging. 1988 Jan;9:9-16. https://doi.org/10.1016/S01974580(88)80004-6

6. Wallace JI, Schwartz RS, LaCroix AZ, Uhlmann RF, Pearlman RA. Involuntary Weight Loss in Older Outpatients: Incidence and Clinical Significance. Journal of the American Geriatrics Society. 1995 Apr;43(4):329-37. https://doi.org/10.1111/j.1532-5415.1995.tb05803.x PMid:7706619

7. Saka B, Kaya O, Ozturk GB, Erten N, Karan MA. Malnutrition in the elderly and its relationship with other geriatric syndromes. Clinical Nutrition. 2010 Dec;29(6):745-8. https://doi.org/10.1016/j.clnu.2010.04.006 PMid:20627486

8. Fried LP, Tangen CM, Walston J, Newman AB, Hirsch C, Gottdiener J, et al. Frailty in Older Adults: Evidence for a Phenotype. The Journals of Gerontology Series A: Biological Sciences and Medical Sciences. 2001 Mar 1;56(3):M146-57. https://doi.org/10.1093/gerona/56.3. m146 PMid:11253156

9. Cruz-Jentoft AJ, Bahat $G$, Bauer J, Boirie $Y$, Bruyère $O$, Cederholm T, et al. Sarcopenia: revised European consensus on definition and diagnosis. Age and Ageing. 2019 Jan 1;48(1):16-31. https://doi.org/10.1093/ageing/ afy169 PMid:30312372 PMCid:PMC6322506

10. Cederholm T, Bosaeus I, Barazzoni R, Bauer J, Van Gossum A, Klek S, et al. Diagnostic criteria for malnutrition - An ESPEN Consensus Statement. Clinical Nutrition. 2015 Jun;34(3):335-40. https://doi.org/10.1016/j.clnu.2015.03. 001 PMid:25799486

11. Evans WJ, Morley JE, Argilés J, Bales C, Baracos V, Guttridge D, et al. Cachexia: A new definition. Clinical Nutrition. 2008 Dec;27(6):793-9. https://doi.org/10.1016/j.clnu.2008.06. 013 PMid: 18718696

12. Cerri AP, Bellelli G, Mazzone A, Pittella F, Landi F, Zambon $A$, et al. Sarcopenia and malnutrition in acutely ill hospitalized elderly: Prevalence and outcomes. Clinical Nutrition. 2015 Aug;34(4):745-51. https://doi.org/10.1016/ j.clnu.2014.08.015 PMid:25263170

13. Frisoli A, Chaves PH, Ingham SJM, Fried LP. Severe osteopenia and osteoporosis, sarcopenia, and frailty status in community-dwelling older women: Results from the Women's Health and Aging Study (WHAS) II. Bone. 2011 Apr;48(4):952-7. https://doi.org/10.1016/j.bone.2010.12. 025 PMid:21195216

14. Boulos C, Salameh P, Barberger-Gateau P. Malnutrition and frailty in community dwelling older adults living in a rural setting. Clinical Nutrition. 2016 Feb;35(1):138-43. https://doi.org/10.1016/j.clnu.2015.01.008 PMid:25649256 
15. Jung H-W, Kim S-W, Kim I-Y, Lim J-Y, Park H-S, Song W, et al. Protein Intake Recommendation for Korean Older Adults to Prevent Sarcopenia: Expert Consensus by the Korean Geriatric Society and the Korean Nutrition Society. Ann Geriatr Med Res. 2018 Dec 31;22(4):167-75. https://doi.org/10.4235/agmr.18.0046 PMid:32743269 PMCid:PMC7387625

16. Sousa-Santos AR, Afonso C, Borges N, Santos A, Padrão P, Moreira $P$, et al. Sarcopenia, physical frailty, undernutrition and obesity cooccurrence among Portuguese communitydwelling older adults: results from Nutrition UP 65 crosssectional study. BMJ Open. 2020 Jun 1;10(6):e033661. https://doi.org/10.1136/bmjopen-2019-033661 PMid:32546486 PMCid: PMC7305469

17. Ligthart-Melis GC, Luiking YC, Kakourou A, Cederholm T, Maier AB, de van der Schueren MAE. Frailty, Sarcopenia, and Malnutrition Frequently (Co-)occur in Hospitalized Older Adults: A Systematic Review and Meta-analysis. Journal of the American Medical Directors Association. 2020 Sep 1 [cited 2020 Oct 13];21(9):1216-28. https://doi. org/10.1016/j.jamda.2020.03.006 PMid:32327302

18. El Okl M, El banouby MH, Mortagy AK. Prevalence of AD and other types of dementia in Egypt. In: International Psychogeriatrics. 2001. p. 114.

19. Wrobel NH, Farrag MF. Preliminary Validation of an Arabic Version of the MMSE in the Elderly. 2007 Mar 3;31(3):75-93. https://doi.org/10.1080/07317110802072223

20. Chaaya M, Sibai A-M, Roueiheb ZE, Chemaitelly H, Chahine $\mathrm{LM}, \mathrm{Al}-\mathrm{Amin} \mathrm{H}$, et al. Validation of the Arabic version of the short Geriatric Depression Scale (GDS-15). IPG. 2008 Jun;20(3):571-81. https://doi.org/10.1017/S104161020800 6741 PMid: 18289453

21. Abd-Al-Atty, M. F, Abou-Hashem, R. M, AbdElaziz, K. M. Prevalence of malnutrition in recently hospitalized elderly in Cairo using a valid and reliable short form of Arabic version of Mini-Nutritional Assessment (MNA-SF-A). Middle East Journal of Age and Ageing. 2012;9(4):8-12. Available at: https://platform.almanhal.com/GoogleScholar/Details/?! $D=2-12944$

22. Vellas B, Guigoz Y, Garry PJ, Nourhashemi F, Bennahum D, Lauque $S$, et al. The mini nutritional assessment (MNA) and its use in grading the nutritional state of elderly patients. Nutrition. 1999 Feb;15(2):116-22. https://doi.org/10.1016/ S0899-9007(98)00171-3

23. Katz S, Downs TD, Cash HR, Grotz RC. Progress in Development of the Index of ADL. The Gerontologist. 1970 Mar 1;10(1 Part 1):20-30. https://doi.org/10.1093/geront/ 10.1_Part_1.20 PMid:5420677

24. Lawton MP, Brody EM. Assessment of older people: selfmaintaining and instrumental activities of daily living. Gerontologist. 1969;9(3):179-86. https://doi.org/10.1093/ geront/9.3_Part_1.179 PMid:5349366

25. Fillenbaum GG, World Health Organization. The wellbeing of the elderly: approaches to multidimensional assessment / Gerda G. Fillenbaum. 1984; Available at: https://apps.who.int/iris/handle/10665/39694

26. Stineman MG, Streim JE, Pan Q, Kurichi JE, SchüsslerFiorenza Rose SM, Xie D. Activity Limitation Stages Empirically Derived for Activities of Daily Living (ADL) and Instrumental ADL in the U.S. Adult Community-Dwelling Medicare Population. PM\&R. 2014 Nov;6(11):976-87. https://doi.org/10.1016/j.pmrj.2014. 05.001 PMid:24798263 PMCid:PMC4216780
27. Charlson M, Szatrowski TP, Peterson J, Gold J. Validation of a combined comorbidity index. Journal of Clinical Epidemiology. 1994 Nov;47(11):1245-51. https://doi.org/ 10.1016/0895-4356(94)90129-5

28. Poe SS, Cvach M, Dawson PB, Straus H, Hill EE. The Johns Hopkins Fall Risk Assessment Tool: Postimplementation Evaluation. Journal of Nursing Care Quality. 2007 Oct;22(4):293-8. https://doi.org/10.1097/01.NCQ.0000290 408.74027.39 PMid:17873724

29. Waldo BR. Grip Strength Testing. Strength \& Conditioning Journal. 1996;18(5). Available at: https://journals.Iww.com /nsca-scj/Citation/1996/10000/Grip_Strength_Testing.5. aspx

30. Podsiadlo D, Richardson S. The Timed "Up \& Go": A Test of Basic Functional Mobility for Frail Elderly Persons. Journal of the American Geriatrics Society. 1991 Feb;39(2):142-8. https://doi.org/10.1111/j.1532-5415.1991.tb01616.x PMid:1991946

31. American Psychiatric Association. Diagnostic and Statistical Manual of Mental Disorders. Fifth Edition. American Psychiatric Association; 2013. https://doi.org/10.1176/appi.books.9780890425596

32. Niederman MS, Mandell LA, Anzueto A, Bass JB, Broughton WA, Campbell GD, et al. Guidelines for the Management of Adults with Community-acquired Pneumonia: Diagnosis, Assessment of Severity, Antimicrobial Therapy, and Prevention. Am J Respir Crit Care Med. 2001 Jun;163(7):1730-54. https://doi.org/10.1164/ajrccm.163.7. at1010 PMid:11401897

33. Rowe TA, Juthani-Mehta M. Diagnosis and Management of Urinary Tract Infection in Older Adults. Infectious Disease Clinics of North America. 2014 Mar;28(1):75-89. https://doi.org/10.1016/j.idc.2013.10.004 PMid:24484576 PMCid:PMC4079031

34. Rasheedy D, El-Kawaly WH. The accuracy of the Geriatric Nutritional Risk Index in detecting frailty and sarcopenia in hospitalized older adults. Aging Clinical and Experimental Research. 2020 Feb 8;32:2469-77. https://doi.org/10.1007/ s40520-020-01492-5 PMid:32036578

35. Cesari M. Physical Frailty and Sarcopenia: Development of a Framework for Supporting Interventions Against Incident Mobility Disability. Ann Geriatr Med Res. 2017 Jun 30;21(2):42-8. https://doi.org/10.4235/agmr.2017.21.2.42

36. Ibrahim K, Howson FFA, Culliford DJ, Sayer AA, Roberts HC. The feasibility of assessing frailty and sarcopenia in hospitalised older people: a comparison of commonly used tools. BMC Geriatr. 2019 Dec;19(1):42. https://doi.org/10.1186/s12877-019-1053-y PMid:30770722 PMCid:PMC6377779

37. Samper-Ternent R, Reyes-Ortiz C, Ottenbacher KJ, Cano CA. Frailty and sarcopenia in Bogotá: results from the SABE Bogotá Study. Aging Clin Exp Res [Internet]. 2017 Apr;29(2):265-72. https://doi.org/10.1007/s40520-01605612 PMid: 27034288

38. Nishiguchi S, Yamada M, Fukutani N, Adachi D, Tashiro Y, Hotta T, et al. Differential Association of Frailty with Cognitive Decline and Sarcopenia in Community-Dwelling Older Adults. Journal of the American Medical Directors Association. 2015 Feb;16(2):120-4. https://doi.org/10.1016/ j.jamda.2014.07.010 PMid:25244957 
39. Bartali B, Frongillo EA, Bandinelli S, Lauretani F, Semba RD, Fried LP, et al. Low Nutrient Intake Is an Essential Component of Frailty in Older Persons. The Journals of Gerontology Series A: Biological Sciences and Medical Sciences. 2006 Jun 1;61(6):589-93. https://doi.org/10.1093/ gerona/61.6.589 PMid:16799141 PMCid: PMC2645617

40. Cruz-Jentoft AJ, Baeyens JP, Bauer JM, Boirie Y, Cederholm T, Landi F, et al. Sarcopenia: European consensus on definition and diagnosis: Report of the European Working Group on Sarcopenia in Older People. Age and Ageing. 2010 Jul 1;39(4):412-23. https://doi.org/10.1093/ageing/afq034 PMid:20392703 PMCid:PMC2886201

41. Vandewoude MFJ, Alish CJ, Sauer AC, Hegazi RA. Malnutrition-Sarcopenia Syndrome: Is This the Future of Nutrition Screening and Assessment for Older Adults? Journal of Aging Research. 2012;2012:1-8. https://doi.org/ 10.1155/2012/651570 PMid:23024863 PMCid:PMC3449123

42. Huang C-Y, Hwang A-C, Liu L-K, Lee W-J, Chen L-Y, Peng L$\mathrm{N}$, et al. Association of Dynapenia, Sarcopenia, and Cognitive Impairment Among Community-Dwelling Older Taiwanese. Rejuvenation Research. 2016 Feb;19(1):71-8. https://doi.org/10.1089/rej.2015.1710 PMid:26165544

43. Sgrò G, Caruso C, Ceravolo F, Curinga G, Renda GF, Rispoli $\mathrm{V}$, et al. Relationship between cognitive impairment and nutritional assessment on functional status in Calabrian long-term-care. CIA. 2014 Jan;9:105-10. https://doi.org/ 10.2147/CIA.S54611 PMid:24453481 PMCid:PMC3892960

44. Persico I, Cesari M, Morandi A, Haas J, Mazzola P, Zambon A, et al. Frailty and Delirium in Older Adults: A Systematic Review and Meta-Analysis of the Literature. J Am Geriatr Soc. 2018;66(10):2022-30. https://doi.org/10.1111/jgs. 15503 PMid:30238970
45. Bellelli G, Zambon A, Volpato S, Abete P, Bianchi L, Bo M, et al. The association between delirium and sarcopenia in older adult patients admitted to acute geriatrics units: Results from the GLISTEN multicenter observational study. Clinical Nutrition. 2018 Oct;37(5):1498-504. https://doi.org/ 10.1016/j.clnu. 2017.08.027

46. Rosted E, Prokofieva T, Sanders S, Schultz M. Serious Consequences of Malnutrition and Delirium in Frail Older Patients. Journal of Nutrition in Gerontology and Geriatrics. 2018 Apr 3;37(2):105-16. https://doi.org/ 10.1080/21551197.2018.1470055 PMid:29792577

47. Mori H, Tokuda Y. Differences and overlap between sarcopenia and physical frailty in older communitydwelling Japanese. Asia Pacific Journal of Clinical Nutrition. 2019 Mar;28(1):157-65. https://doi.org/10.6133/ apjcn.201903_28(1).0021

48. Kadam U, Croft P, North Staffordshire GP Consortium Group. Clinical multimorbidity and physical function in older adults: a record and health status linkage study in general practice. Family Practice. 2007 Aug 14;24(5):412-9. https://doi.org/10.1093/fampra/cmm049 PMid:17698977

49. Wolff JL, Starfield B, Anderson G. Prevalence, Expenditures, and Complications of Multiple Chronic Conditions in the Elderly. Arch Intern Med. 2002 Nov 11;162(20):2269. https://doi.org/10.1001/archinte.162.20.2269 PMid:12418941 


\section{APPENDIX}

\section{Current Recommendation Criteria to Define Tissue Loss Syndromes (Physical Frailty, Sarcopenia, Cachexia, and} Malnutrition)

\begin{tabular}{|c|c|}
\hline Component & Definition \\
\hline $\begin{array}{l}\text { Physical frailty } \\
\text { According to the criteria proposed by } \\
\text { Fried et al. [8] }\end{array}$ & 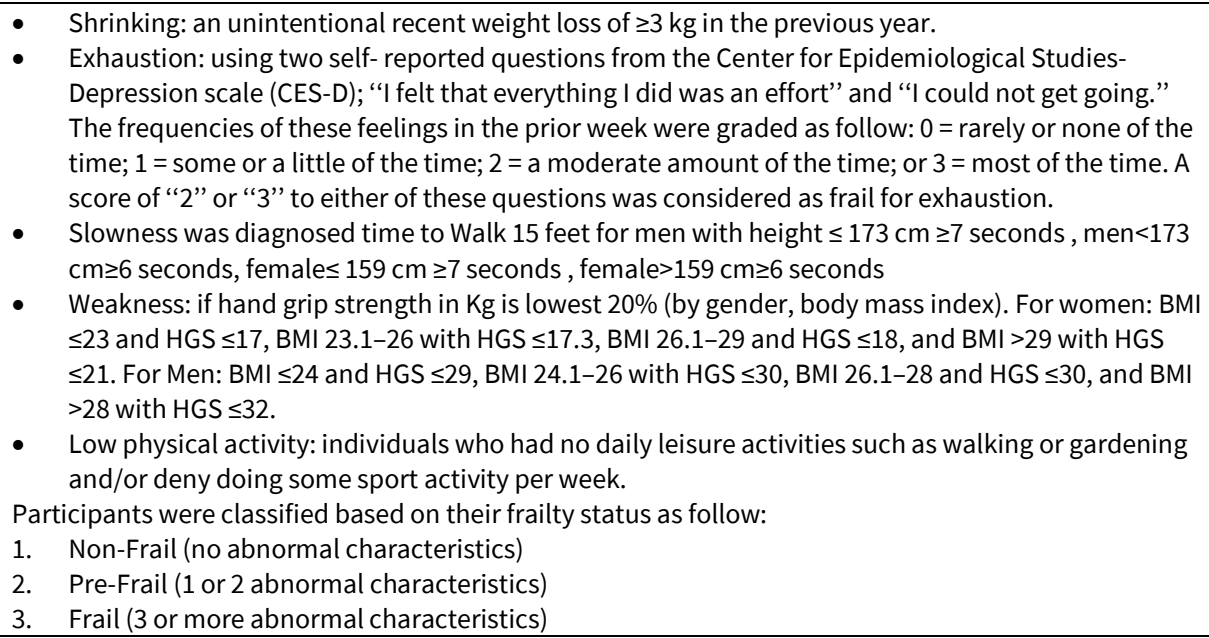 \\
\hline $\begin{array}{l}\text { Sarcopenia } \\
\text { According to according to the recent } \\
\text { update EWGSOP [9] }\end{array}$ & $\begin{array}{l}\text { - } \\
\text { Probable sarcopenia if low muscle strength is detected (HGS }<27 \mathrm{~kg} \text { for men and }<16 \mathrm{~kg} \text { for } \\
\text { - } \quad \text { Confirmed sarcopenia diagnosis by additional documentation of low muscle quantity/quality } \\
\text { (Appendicular Skeletal Muscle Mass adjusted for height squared (ASM/height } 2 \text { ) }<7.0 \mathrm{~kg} / \mathrm{m} 2 \mathrm{for} \\
\text { men and }<5.5 \mathrm{~kg} / \mathrm{m} 2 \text { for women(SMI) } \\
\text { - Severe sarcopenia if low muscle strength, low muscle quantity/quality and low physical } \\
\text { performance (TUG test } \geq 20 \mathrm{~s} \text { ) are all documented. } \\
\text { While, pre-sarcopenia is defined as low SMI only }\end{array}$ \\
\hline $\begin{array}{l}\text { Cachexia } \\
\text { According to criteria proposed by } \\
\text { Evans et al. [11] }\end{array}$ & $\begin{array}{l}\text { (A) Weight loss of at least } 5 \% \text { in } 12 \text { months or less in the presence of an underlying illness } \\
\text { (B) Three of the following five criteria: } \\
\text { 1. Decreased muscle strength } \\
\text { 2. Fatigue } \\
\text { 3. Anorexia } \\
\text { 4. Low fat-free mass index } \\
\text { 5. Abnormal biochemistry: } \\
\text { a. Increased inflammatory markers }(\mathrm{CRP}, \mathrm{IL}-6) \\
\text { b. Anemia }(\mathrm{Hb}<12 \mathrm{~g} / \mathrm{dl}) \\
\text { c. Low serum albumin }(<3.2 \mathrm{~g} / \mathrm{dl})\end{array}$ \\
\hline $\begin{array}{l}\text { Malnutrition } \\
\text { According to the ESPEN } \\
\text { Consensus Statement [10] }\end{array}$ & $\begin{array}{l}\text { Those with MNA SF score below } 12 \text { with either alternative } 1 \text { or } 2 \\
\text { Alternative } 1 \text { : BMI }<18.5 \mathrm{~kg} / \mathrm{m}^{2} \\
\text { Alternative } 2 \text { : Weight loss (unintentional) }>10 \% \text { indefinite of time, or }>5 \% \text { over the last } 3 \text { months } \\
\text { combined with either } \\
\text { - } \quad \mathrm{BMI}<20 \mathrm{~kg} / \mathrm{m}^{2} \text { if }<70 \text { years of age, or }<22 \mathrm{~kg} / \mathrm{m}^{2} \text { if } \geq 70 \text { years of age or } \\
\text { - } \quad \mathrm{FFMl}<15 \text { and } 17 \mathrm{~kg} / \mathrm{m}^{2} \text { in women and men, respectively. }\end{array}$ \\
\hline
\end{tabular}

